\title{
DETERMINANTAL INEQUALITIES OF POSITIVE DEFINITE MATRICES
}

\section{DAESHIK CHOI}

Abstract. Let $A_{i}, i=1, \ldots, m$, be positive definite matrices with diagonal blocks $A_{i}^{(j)}, 1 \leqslant j \leqslant$ $k$, where $A_{1}^{(j)}, \ldots, A_{m}^{(j)}$ are of the same size for each $j$. We prove the inequality

$$
\operatorname{det}\left(\sum_{i=1}^{m} A_{i}^{-1}\right) \geqslant \operatorname{det}\left(\sum_{i=1}^{m}\left(A_{i}^{(1)}\right)^{-1}\right) \cdots \operatorname{det}\left(\sum_{i=1}^{m}\left(A_{i}^{(k)}\right)^{-1}\right)
$$

and more determinantal inequalities related to positive definite matrices.

Mathematics subject classification (2010): 15A45.

Keywords and phrases: Determinantal inequalities, Fischer's inequality, determinants of block matrices, positive definite matrices.

\section{REFERENCES}

[1] R. Horn And C. Johnson, Matrix Analysis, Cambridge University Press, 1990.

[2] M. Lin, Determinantal inequalities for block triangular matrices, Math. Inequal. Appl., Volume 18, Number 3 (2015), 1079-1086.

[3] I. Matic, Inequalities with determinants of perturbed positive matrices, Linear Algebra Appl., Volume 449, 2015, 166-174. 